\title{
Sudden Cardiac Death (SCD) Prediction based on Fast Sequency Ordered Complex Hadamard Transform
}

\author{
Padmavathi Kora \\ Gokaraju Rangaraju Institute of Engineering Technology \\ Hyderabad \\ India
}

\begin{abstract}
Electrocardiogram (ECG), a non-invasive diagnostic technique, used for detecting cardiac arrhythmia has gained attention in recent years in medical sciences, industry dealing with Bio-medical instrumentation and research, demanding an advancement in its ability to distinguish different cardiac arrhythmia. Studies conducted in this research work on recent feature extraction methods, such as, Auto Regressive (AR) modeling, Magnitude Squared Coherence (MSC) and Wavelet Coherence (WTC) using standard database (MIT-BIH), also yielded a lot of features. A large number of these features might be insignificant containing some redundant and non-discriminative features that introduce computational burden and loss of performance. A novel technique to classify the ECGs of normal and subjects at risk of SCD using nonlinear technique has been presented. We have predicted SCD by analyzing four minutes of ECG signals prior to SCD occurrence by using Fast CS-SCHT coefficients. This paper presents fast Conjugate Symmetric Sequency Ordered Complex Hadamard Transform (CS-SCHT) for extracting relevant features from the ECG signal. The sparse matrix factorization method is used for developing fast and efficient CS-SCHT algorithm and its computational burden is examined as compared to that of the HT and NCHT. The applications of the CS-SCHT in the ECG based SCD detection is also discussed. In this work, we have achieved good classification accuracy for prediction of SCD. The proposed method is able to detect a person at risk of SCD four minutes earlier.
\end{abstract}

\section{General Terms}

Sudden Cardiac Death (SCD) Prediction based on Fast Sequency Ordered Complex Hadamard Transform

\section{Keywords}

Sudden Cardiac Death, ECG, Fast CS-SCHT, Neural Network Classifier

\section{INTRODUCTION}

A surprising sudden death of a patient with or without essential cardiovascular difficulties and known or unknown history of car- diovascular illnesses is classified as Sudden Cardiac Death (SCD). The general yearly frequency of SCD taking into account extrapolation of information from the United States is roughly 1 in 1000 grown-ups over the age of 35 years. SCD is the most well-known and by and large the essential sign of coronary illness and is responsible for the majority of the cardiovascular death in the US and other developing nations. Despite the fact that, the main source of SCD is unclear, Ventricular Fibrillation (VF) is assumed to be the mechanism roughly $20 \%$ SCD episodes. VF can prompt the heart's inability to successfully pump blood and in this way can bring about death in few minutes. Hence, early expectation of a sudden SCD problem in a patient encountering VF is highly important for intime treatment and expanded survival rate.

Scientists worldwide are exploring different techniques for ECG in order to get the fine changes happening inside the signals and to recognize the unique highlights which can caution the danger of SCD onset. In this study, computation of QT interval prolongation is appeared to be an independent risk component to anticipate SCD because of heart failure. It has been demonstrated that straight forecast techniques can be utilized for examination of heart rate variability signs, QT scattering, signal averaged ECG (SAECG) and QRS length, which can viably be utilized as risk measure for foreseeing the event of SCD after Acute Myocardial Infarction (AMI) [14] in Type 2 diabetic patients. This paper aims at developing an intelligent, inexpensive and flexible ECG-based automatic arrhythmia (irregular heart beat) detection system. The method includes signal processing and feature extraction techniques to obtain the discriminative features of the ECG signals that correspond to a cardiac condition. These ECG features are classified using supervised learning techniques.

Various algorithms for the feature extraction of ECG signal are like Haar transform (Hat) [10], the Karhunen-Loeve transform (KLT) [8], Wavelet Transform (WT) and the Discrete Cosine Transform (DCT) [5], [9]. The Fast Fourier Transform (FFT) performs signal transformation from time to frequency domain for some of the applications [6]. The DCT is widely held for its optimum energy compaction property because the division of the average energy of the signal is grouped into a comparatively a small number of constituents of the DCT-coefficients. The DWT [12] is familiar for its analysis for image and signal processing in multi-resolutional pattern. In the real time applications advanced fast algorithm like Hadamard Transform (HT) [2] is used. Advantage of applying CSSCHT over HT is its very low computational complexity of order $\log _{2} M$ for M data samples. The BIFORE (Binary Fourier Repre- 

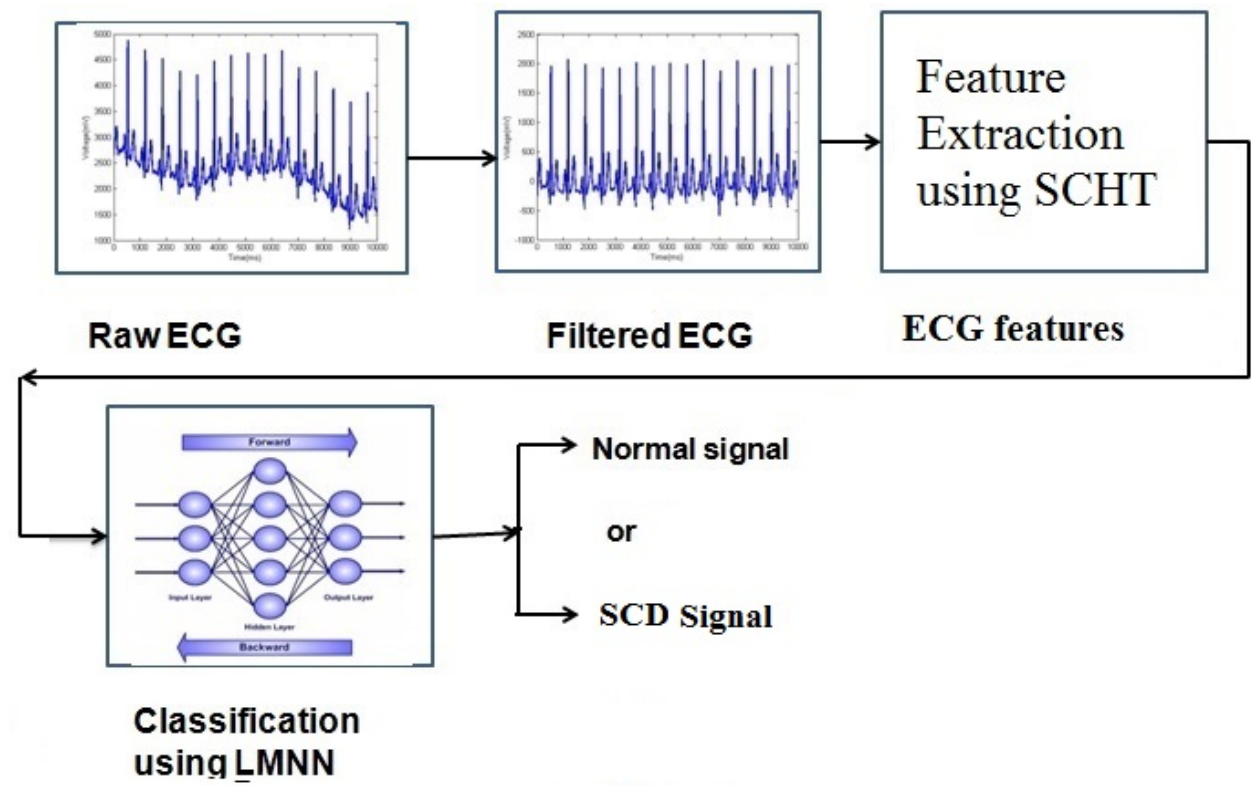

Fig. 2. ECG classification using CS-SCHT features

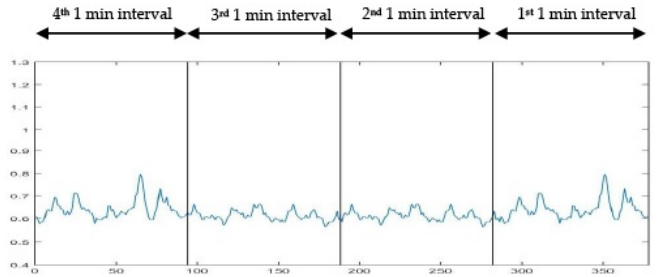

(a)

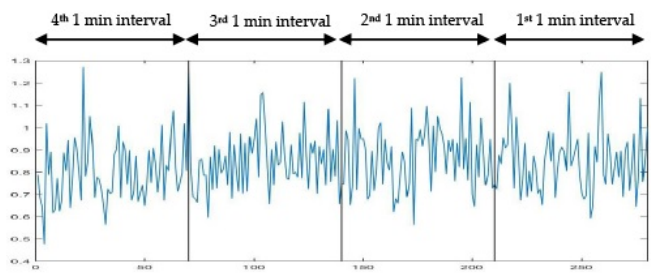

(b)

Fig. 1. Four one minute intervals of (a) normal and (b) SCD ECG signal.

sentation) [1] or HADAMARD transform contains two levels $( \pm)$. It is used in various applications like signal processing, data compression algorithms, data encryption, quantum computing, etc. But the disadvantage of using this real Hadamard transform is that it can be applied only to real values. This limitation can be avoided by using Complex Hadamard Transform (CHT). CHT is a fourvalued $( \pm 1, \pm \mathrm{j})$ sequence. CHT includes high ordered matrices and Kronecker products. CHT with sequency order is called SCHT. Sequency measures how fast the elements of a specific row vector of the Hadamard transform matrix vary over a normalized time base $t \epsilon[0,1]$. SCHT coefficients are complex numbers comprising of both real \& imaginary parts and they are not conjugate symmetric. Hence, more memory is needed to store the coefficients for analysis and synthesis in transform implementation. Hence a novel ver- sion of SCHT which surpasses the previous one called Conjugate Symmetric Sequency Ordered Complex Hadamard Transform (CSSCHT) whose spectrum is conjugate symmetric, is projected in this paper. As CS-SCHT spectrum is conjugate symmetric, only half of spectral coefficients are required for combination and investigation. This in turn shrinks the memory requirement in processing for the applications such as real-time image watermarking and spectrum estimation.

\section{PRE-PROCESSING}

SCD Holter open access database and traditional Normal Sinus Rhythm (NSR) information were acquired from the SCD and NSR database of MIT-BIH database. A complete of forty-one cardiogram signals of twenty three SCD patients (age: eighteen- 89 years) and 18 traditional subjects (20-50 years) were taken. Among the twenty three SCD patients, solely twenty were used additional for analysis, as they did not exhibit any episodes of VF. The rate of SCD and traditional cardiogram signals were 256 and $128 \mathrm{~Hz}$ respectively. The signals were sampled at 128 cycle so as to maintain consistency between SCD and normal. From the twenty four hours of SCD patientsâĂŹ ECG recordings, only the ECG signals four minutes before the SCD occurrence are used. From the twenty four hours graph recordings, SCD signals of ten minutes measure extracted. The obtained SCD signals of 4 minutes length square measure any divided into four one minute intervals. the small print of this method used for graph signal is discussed in our previous paper. The signals obtained are then subjected SCHT for feature extraction.

\section{FEATURE EXTRACTION}

Feature extraction is the important step in the detection of heart arrhythmia. Each ECG beat consists of large number of features and many of them might be insignificant. In this paper CS-SCHT extracts the features of each cardiac beat. 


\subsection{Conjugate Symmetric-Sequency Ordered Complex Hadamard Transform (CS-SCHT)}

The row vectors of real Hadamard matrices can be arranged in a number of ways to obtain the ordered Hadamard matrices. Sequency order is widely preferred due to its analogy to Fourier transform and its applicability in digital signal processing and communications. Sequency measures how fast the elements of a specific row vector of the Hadamard transform matrix vary over a normalized time base $\mathrm{k} \epsilon[0,1)$. Hence, it is also interesting to explore the orderings of complex Hadamard transforms for some particular applications. Complex Hadamard Transform (CHT) with a sequency order known as Sequency-Ordered Complex Hadamard Transform (SCHT) is presented. Complex plane within the normalized time $t$, that is The construction of the SCHT matrices is based on the complex Rademacher functions which are the extended complex version of conventional Rademacher functions. The row vectors of an SCHT matrix are arranged in an increasing order of zero crossings in the unit circle of a, $0 \leq k \geq 1$.

\subsection{Fast CS-SCHT Algorithm using Factorization method}

Let $H_{M}$ be any CS-SCHT [3] matrix of size $\mathrm{M} \mathrm{x} \mathrm{M}$. Then, it is a square matrix defined by

$$
H_{M}=\left[\begin{array}{cc}
H_{M / 2} & H_{M / 2} \\
H_{M / 2} S_{M / 2} & -H_{M / 2} S_{M / 2}
\end{array}\right] \cdot P_{m}
$$

where $M=2^{m}$ and $P_{m}$ is the permutation matrix.

$$
S_{2^{m-1}}=\left[\begin{array}{cc}
I_{2^{m-2}} & 0 \\
0 & j I_{2^{m-2}}
\end{array}\right]
$$

and $I_{2^{m-2}}$ is the identity matrix of order $2^{m-2} \times 2^{m-2}$. In this way, an M x M CS-SCHT matrix can be constructed using the CS-SCHT matrices of size $(\mathrm{M} / 2) \times(\mathrm{M} / 2)$, and the CS-SCHT matrix of order $8 \mathrm{x} 8$, which is expressed as

$$
H_{8}=\left[\begin{array}{cc}
H_{4} & H_{4} \\
H_{4} S_{4} & -H_{4} S_{4}
\end{array}\right] \cdot P_{m}
$$

A well-organized algorithm for calculating the CS-SCHT is formulated using the sparse-matrix factorization method. In order to understand the factorization method, for $\mathrm{M}=8$, then $H_{8}$ matrix will become

$$
H_{8}=\left[\begin{array}{cc}
H_{4} & 0 \\
0 & H_{4}
\end{array}\right]\left[\begin{array}{cc}
I_{4} & 0 \\
0 & S_{4}
\end{array}\right]\left[\begin{array}{cc}
I_{4} & I_{4} \\
I_{4} & -I_{4}
\end{array}\right] \cdot P_{m}
$$

$\mathrm{H}_{4}$ can be again factorized and represented in terms of $\mathrm{H}_{2}$ as,

$$
\begin{aligned}
& H_{8}=\left[\begin{array}{cccc}
H_{2} & 0 & 0 & 0 \\
0 & H_{2} & 0 & 0 \\
0 & 0 & H_{2} & 0 \\
0 & 0 & 0 & H_{2}
\end{array}\right] \cdot\left[\begin{array}{cccc}
I_{2} & 0 & 0 & 0 \\
0 & S_{2} & 0 & 0 \\
0 & 0 & I_{2} & 0 \\
0 & 0 & 0 & S_{2}
\end{array}\right] . \\
& {\left[\begin{array}{cccc}
I_{2} & I_{2} & 0 & 0 \\
I_{2} & -I_{2} & 0 & 0 \\
0 & 0 & I_{2} & I_{2} \\
0 & 0 & I_{2} & -I_{2}
\end{array}\right] \cdot\left[\begin{array}{cc}
I_{4} & 0 \\
0 & S_{4}
\end{array}\right] \cdot\left[\begin{array}{cc}
I_{4} & I_{4} \\
I_{4} & -I_{4}
\end{array}\right] \cdot P_{m}}
\end{aligned}
$$

where

$$
H_{2}=\left[\begin{array}{cc}
1 & 1 \\
1 & -1
\end{array}\right], S_{2}=\left[\begin{array}{ll}
1 & 0 \\
0 & j
\end{array}\right], S_{4}=\left[\begin{array}{cc}
I_{2} & 0 \\
0 & j I_{2}
\end{array}\right]
$$

and $P_{m}$ is the permutation matrix.

Decomposition of 8-point CS-SCHT [4] into three stages as shown in Figure 5 Each stage requires eight subtractions/additions. Apart from first stage remaining stages involves complex multiplications by $\mathrm{j}$ to compute the 8-point CS-SCHT. Total mathematical requirements needed are $(M / 4) \log _{2}(M / 2)$ complex multiplications and $M \log _{2} M$ complex addition/subtractions, where $M=2^{m}$ and $\mathrm{m}$ is the number of stages. The hardware implementation of the complex mathematical computations can be obtained by swapping and negation operations. In similar procedure $H_{128}$ matrix is generated. Each ECG beat of 128 samples is multiplied with CS-SCHT matrix of order $128 \mathrm{X} 128$ in order to transform it into Hadamard domain.

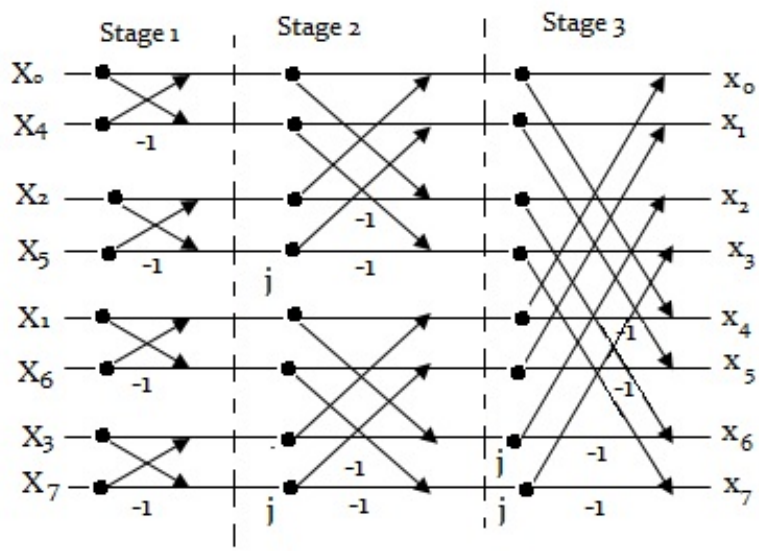

Fig. 3. Fast implementation of CS-SCHT

\section{RESULTS AND DISCUSSION}

The MIT-BIH SCD Holter open access database and the Normal Sinus Rhythm database were used to obtain the SCD and normal ECG signals. A total of 41 ECG signals of 23 SCD patients (age: $18-89$ years) and 18 normal subjects (20-50 years) were acquired from the database. Among the 23 SCD patients, only 20 were used further for analysis, as they did not exhibit any episodes of VF. The sampling rate of SCD and normal ECG signals were $256 \mathrm{~Hz}$ and $128 \mathrm{~Hz}$, respectively. All the ECG signals were re-sampled at $128 \mathrm{~Hz}$ in order to maintain consistency between SCD and normal groups in this work.

For this work, from the 24 hours of SCD patientsâĂŹ ECG recordings, only the ECG signals four minutes before the SCD occurrence (onset) are used. The ECG signals obtained are then subjected to wavelet based denoising using daubechies $6(\mathrm{db} 6)$ mother wavelet. From the 24 hours ECG recordings, SCD signals of 10 minutes are extracted and only the four minutes duration SCD is used for further analysis. The obtained SCD signals of four minutes duration are further divided into four one minute intervals. The algorithm has been implemented in Matlab 7.12.0. The experiment has been employed with a population size of 2086. In this paper CS-SCHT is used as the feature extraction technique. The CS-SCHT gives best features for the classification. The performance of CS-SCHT is compared with classical HT, NCHT techniques. The HT, NCHT 
and CS-SCHT features are classified using SVM, KNN and LM NN as in the Tables 1, 2, 3. For measuring accuracy two parameters Sensitivity (Sen) and Specificity (Spe) are calculated using the following equations.

$$
\begin{gathered}
\text { Spe }=\frac{\text { Coreectly_classified_Normal }- \text { beats }}{\text { Total_Normal_beats }} X 100 \\
\text { Sen }=\frac{\text { Correctly_classified_SCD_beats }}{\text { Total_SCD_beats }} X 100 \\
\text { Accuracy }=\frac{\text { Coreectly_classified_beats }}{\text { Total_beats }} X 100
\end{gathered}
$$

Table 1.

Classification

with

SVM

clas-

si-

fier

\begin{tabular}{cccc}
\hline Classifier & Sen & Spe & Accuracy \\
\hline HT+KNN & $53.2 \%$ & $65.1 \%$ & $51.2 \%$ \\
NCHT+KNN & $73.5 \%$ & $72.2 \%$ & $73.22 \%$ \\
Fast CS-SCHT+KNN & $92.35 \%$ & $93.9 \%$ & $92.17 \%$ \\
\hline
\end{tabular}

Table 2.

Classification

with

SVM

clas-

si-

fier

\begin{tabular}{cccc}
\multicolumn{5}{c}{ fier } & & \\
\hline Classifier & Sen & Spe & Accuracy \\
\hline HT+SVM & $71.0 \%$ & $73.13 \%$ & $70.12 \%$ \\
NCHT+SVM & $76.2 \%$ & $75.47 \%$ & $72.13 \%$ \\
Fast CS-SCHT+SVM & $95.5 \%$ & $96.9 \%$ & $96.74 \%$ \\
\hline
\end{tabular}

Table 3.

\begin{tabular}{cccc} 
& $\begin{array}{l}\text { Table } 3 . \\
\text { Classification } \\
\text { with }\end{array}$ & \\
& LM & & \\
& NN & & \\
& clas- & & \\
& si- & & \\
& fier & & \\
\hline & Sen & Spe & Accuracy \\
\hline Classifier & $91.2 \%$ & $89.2 \%$ & $80.9 \%$ \\
HT+LMNN & $89.34 .2 \%$ & $89.2 \%$ & $89.2 \%$ \\
NCHT+LMNN & $99.97 \%$ & $98.7 \%$ & $99.3 \%$ \\
\hline
\end{tabular}

Figure 4 [and 6 compare the accuracy performance of KNN, SVM and LMNN classifiers in terms of ROC curves. The classification accuracy of KNN classifier with CS-SCHT optimized features is $92.17 \%$ for the detection of SCD. The classification accuracy of SVM classifier with CS-SCHT optimized features is $96.74 \%$ for

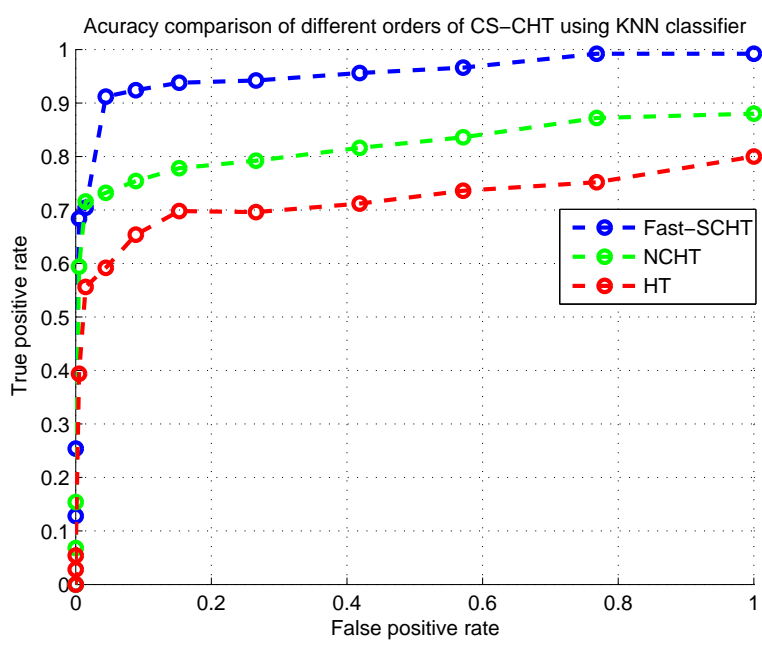

Fig. 4. Performance comparison of different orders of Hadamard Transform

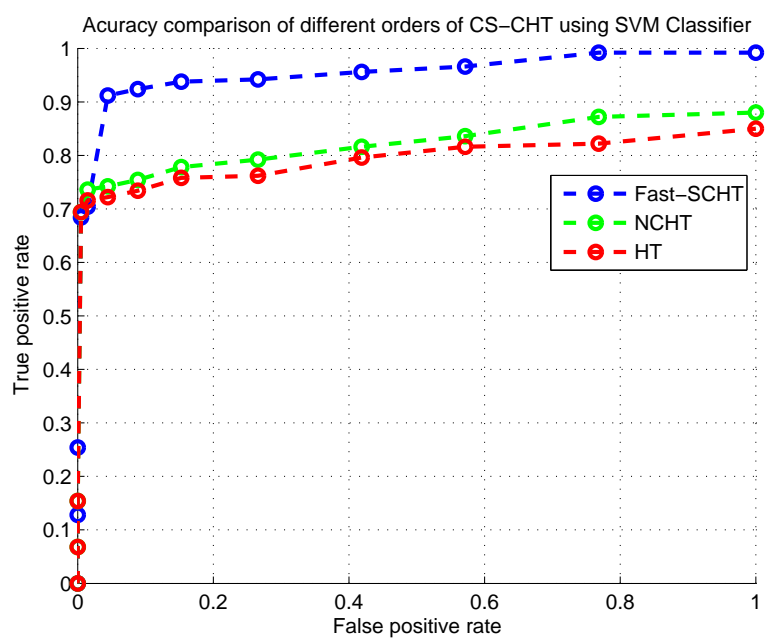

Fig. 5. Performance comparison of different orders of Hadamard Transform

the detection of SCD. Results show that optimized CS-SCHT features in combination with LMNN classifier shows better results than KNN and SVM classifiers for the detection of SCD. The classification accuracy of LMNN classifier with CS-SCHT features is 99.3\% for the detection of SCD. Voss et al. [14] used frequency and time domain features of 26 cardiac patients after MI using nonlinear methods and renormalized entropy. They observed $96 \%$ classification using the combination of all features. Shen et al. [13] developed a personal cardiac model for SCD detection from ECGs. They observed classification accuracy of $87.5 \%$ using wavelets for the detection of SCD. Elias et al. [7] in their study developed a novel approach to predict SCD one, two, three and four minutes before its onset using nonlinear and TF analysis of heart rate variability signals. The algorithm extracted time-frequency features of average energy and nonlinear features of Poincare plot and non- 
Table 4.

Comparative

study

for

de-

tec-

tion

of

SCD

\begin{tabular}{|c|c|c|c|c|}
\hline Studies & Approach & Sen $(\%)$ & Spe (\%) & $\operatorname{Acc}(\%)$ \\
\hline Shen et al. (2007) [13] & $\begin{array}{l}\text { Wavelet analysis (db2) and } \\
\text { HRV analysis using FFT }\end{array}$ & 75 & - & 87.5 \\
\hline Voss et al. (1996) [14] & $\begin{array}{l}\text { Linear and Nonlinear dy- } \\
\text { namic methods }\end{array}$ & - & - & 96 \\
\hline Manis et al. (2013) [11] & $\begin{array}{l}\text { Linear and Nonlinear fea- } \\
\text { tures }\end{array}$ & - & - & 87.5 \\
\hline Elias et al. (2014) & $\begin{array}{l}\text { Linear time domain, linear } \\
\text { frequency domain features }\end{array}$ & 99.64 & 98.27 & 99.03 \\
\hline Proposed approach & Fast CS-SCHT & 96.97 & 99.2 & 99.3 \\
\hline
\end{tabular}

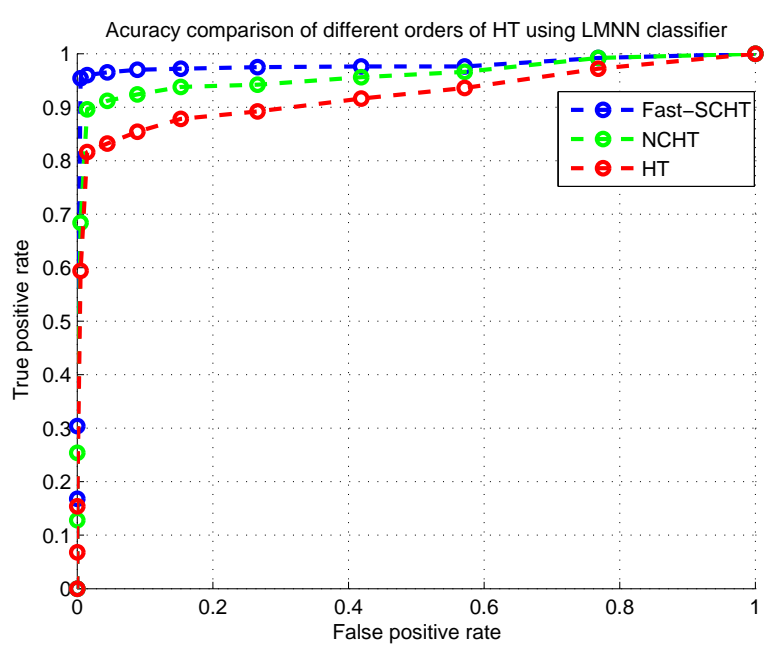

Fig. 6. Performance comparison of different orders of Hadamard Transform

linear short-term fractal scaling exponent from heart rate variability signal and used MLP and KNN classifiers to predict the patients with SCD. Manis et al. [11] designed a machine learning technique to predict high and low risk SCD signals after arrhythmia in heart patients using HRV analysis. They obtained a classification accuracy of $87.5 \%$ by using SVM, and $85 \%$ by using Random Forest classifiers for the detection of SCD. The proposed method shows the highest classification accuracy for the detection of SCD. The CS-SCHT have been employed intelligently to select the most relevant features that could increase the classification accuracy while ignoring noisy and redundant features.

\section{CONCLUSION}

ECG is used to access the electrical activity of a human heart. In this study our aim is to automate the above procedure so that it leads to correct diagnosis. Early diagnosis and treatment is of great importance because immediate treatment can save the life of the patient. The proposed fast CS-SCHT method is used to extract the features from each ECG beat then these features are compared to HT, NCHT algorithm. The classification accuracy using CS-SCHT with LMNN classifier was $99.3 \%$ for the detection of SCD. The experimental results have shown that the proposed CS-SCHT method can extract more relevant features than the other methods proposed in the literature with highest classification accuracy for the detection of SCD. 


\section{REFERENCES}

[1] Nasir Ahmed, KR Rao, and AL Abdussattar. Bifore or hadamard transform. Audio and Electroacoustics, IEEE Transactions on, 19(3):225-234, 1971.

[2] A Aung and BP Ng. Fast dft implementation using scht. Nanyang Technological Univ., Singapore, 2007.

[3] Aye Aung and Boon Poh Ng. Conjugate symmetric sequency ordered complex hadamard transform. IEEE Transactions on Signal Processing, 57(7):2582-2593, 2009.

[4] Aye Aung, Boon Poh Ng, and Susanto Rahardja. Sequencyordered complex hadamard transform: Properties, computational complexity and applications. Signal Processing, IEEE Transactions on, 56(8):3562-3571, 2008.

[5] Kenneth G Beauchamp. Applications of Walsh and related functions. Academic press, 1984.

[6] RH Clayton and A Murray. Estimation of the ecg signal spectrum during ventricular fibrillation using the fast fourier transform and maximum entropy methods. In Computers in Cardiology 1993, Proceedings., pages 867-870. IEEE, 1993.

[7] Elias Ebrahimzadeh, Mohammad Pooyan, and Ahmad Bijar. A novel approach to predict sudden cardiac death (scd) using nonlinear and time-frequency analyses from hrv signals. PloS one, 9(2):e81896, 2014.

[8] Michelle Effros, Hanying Feng, and Kenneth Zeger. Suboptimality of the karhunen-loeve transform for transform coding. Information Theory, IEEE Transactions on, 50(8):16051619, 2004.

[9] Hamid Khorrami and Majid Moavenian. A comparative study of dwt, cwt and det transformations in ecg arrhythmias classification. Expert systems with Applications, 37(8):5751-5757, 2010.

[10] Jose A Rosendo Macias and Antonio Gomez Exposito. Efficient computation of the running discrete haar transform. Power Delivery, IEEE Transactions on, 21(1):504-505, 2006.

[11] George Manis, Stavros Nikolopoulos, Petros Arsenos, Konstantinos Gatzoulis, Polychronis Dilaveris, and Christodoulos Stefanadis. Risk stratification for arrhythmic sudden cardiac death in heart failure patients using machine learning techniques. In Computing in Cardiology 2013, pages 141-144. IEEE, 2013.

[12] C Saritha, V Sukanya, and Y Narasimha Murthy. Ecg signal analysis using wavelet transforms. Bulg. J. Phys, 35(1):6877, 2008.

[13] Tsu-Wang Shen, Hsiao-Ping Shen, Ching-Heng Lin, and YiLing Ou. Detection and prediction of sudden cardiac death (scd) for personal healthcare. In 2007 29th Annual International Conference of the IEEE Engineering in Medicine and Biology Society, pages 2575-2578. IEEE, 2007.

[14] A Voss, J Kurths, HJ Kleiner, A Witt, N Wessel, P Saparin, KJ Osterziel, R Schurath, and R Dietz. The application of methods of non-linear dynamics for the improved and predictive recognition of patients threatened by sudden cardiac death. Cardiovascular research, 31(3):419-433, 1996. 